\title{
Critical parameters and pair correlations in confined multicomponent liquids
}

\author{
A.N.Vasil'ev ${ }^{1}$, A.V.Chalyi ${ }^{1,2}$ \\ 1 Taras Shevchenko Kiev University, \\ Physics Department, \\ 2 Glushkov Prosp., Building 1, Kyiv 03680, Ukraine \\ 2 Department of Physics, \\ National Medical University, \\ 13 Shevchenko Blvd., Kiev 01601, Ukraine
}

Received August 3, 2005, in final form February 20, 2006

We investigate multicomponent finite-size liquid systems with geometry of plane-parallel layer and cylinder. We consider these systems are being affected by weak external field which causes their anisotropy. For these systems we find pair correlation functions and analyze the effect of space limitation and external field on correlative behaviour of multicomponent liquid. We compare our results with the data for simple and binary finite-size systems.

Key words: correlation function, critical state, multicomponent liquid

PACS: $05.70 . F h, 05.70 . J k, 64.60 . F r, 68.35 . R h$

\section{Introduction}

The main point of this paper is to study the critical correlative behavior of finite-size multicomponent liquids. First of all, in spite of the availability of plenty of data on critical behavior of simple [1-4] and binary liquid systems [1,5-8], multicomponent mixtures (even spatially infinite) have not been studied well enough at the moment (for most remarkable results one can see for example [5,9-13]). Moreover, we lack data on the critical correlative behavior of finite-size multicomponent systems. On the other hand, these systems are of great practical importance as well as very interesting from theoretical point of view.

Here we consider a multicomponent liquid system with the geometry of plane-parallel layer and cylinder. External field is applied to these systems. We find pair correlation functions of density fluctuations. Based on these expressions we analyze critical behavior of the system. Namely, we show that space limitation causes the shift of critical parameters and find how these shifts depend on the linear size of the system.

\section{Formalization of the problem}

To find pair correlation functions $G_{i j}(\vec{r})(i, j=1,2, \ldots, N, N$ is the number of mixture components) of density fluctuations we use Ornstein-Zernike (OZ) system of intergral equations. This system can be written in matrix form as follows [14]

$$
\hat{G}(\vec{r})=\hat{F}(\vec{r})+\int \hat{F}\left(\vec{r}_{1}\right) \hat{G}\left(\vec{r}-\vec{r}_{1}\right) \mathrm{d} \vec{r}_{1}
$$

where $\hat{G}(\vec{r})$ is matrix of pair correlation functions (its components are functions $G_{i j}(\vec{r})$ ) and $\hat{F}(\vec{r})$ is matrix of direct correlation functions $F_{i j}(\vec{r})$. All the functions are normalized by average densities $[14]$. 
In the general case of infinite isotropic system, correlation functions depend only on the distance between correlating points $[1,2,15,16]$. In our situation, the system is of finite-size and is affected by the external field. For the sake of simplicity we consider correlations with respect to the point in the middle (in geometrical sense) of the system. This allows us to consider the correlation functions to have the only argument but nevertheless we should suppose that correlation functions depend on the direction as well. That is why in equation (1) we have used vector arguments for correlation functions.

From practical point of view it is more convenient to use differential OZ system of equations. In a matrix form, this system of equations is of the form [14]

$$
\hat{G}(\vec{r})=\hat{F}(\vec{r})+\hat{C}_{(0)} \hat{G}(\vec{r})-\sum_{\alpha=x, y, z} \hat{C}_{(1, \alpha)} \frac{\partial \hat{G}(\vec{r})}{\partial \alpha}+\hat{C}_{(2)} \triangle \hat{G}(\vec{r}),
$$

and here we have defined for the matrices of spatial moments of direct correlation functions

$$
\hat{C}_{(n)}=\frac{1}{(n+1) !} \int \hat{F}(\vec{r}) r^{n} \mathrm{~d} \vec{r}
$$

for $n=0,2$ and

$$
\hat{C}_{(1, \alpha)}=\int \hat{F}(\vec{r}) \alpha \mathrm{d} \vec{r}
$$

$\alpha=x, y, z$. The presence of nonzero first spatial moment is caused by the external field. The solution of matrix equation (2) differs for the system of different geometries. As was mentioned above, we are considering finite-size systems with geometry of plane-parallel layer and infinite cylinder. In both these cases let us assume the external field to be directed along z-axis. In case of a cylinder, z-axis coincides with the main axis of the cylinder and in case of a plane-parallel layer, z-axis is perpendicular to the plane of the layer. This means that $\hat{C}_{(1, x)}=\hat{C}_{(1, y)}=0$ and equation (2) can be rewritten as follows:

$$
\hat{G}(\vec{r})=\hat{F}(\vec{r})+\hat{C}_{(0)} \hat{G}(\vec{r})-\hat{C}_{(1)} \frac{\partial \hat{G}(\vec{r})}{\partial z}+\hat{C}_{(2)} \triangle \hat{G}(\vec{r}),
$$

and we have changed for simplicity $\hat{C}_{(1, z)} \equiv \hat{C}_{(1)}$. If we assume the matrix of pair correlation functions to be of the form

$$
\hat{G}(\vec{r})=\hat{g}(r) \exp (\hat{\gamma} z)
$$

and apply

then we have

$$
\hat{\gamma}=\frac{1}{2} \hat{C}_{(2)}^{-1} \hat{C}_{(1)}
$$

$$
\triangle \hat{g}(r)-\hat{C}_{(2)}^{-1}\left(\hat{E}-\hat{C}_{(0)}+\frac{1}{4} \hat{C}_{(2)}^{-1} \hat{C}_{(1)}^{2}\right) \hat{g}(r)=-\hat{C}_{(2)}^{-1} \hat{f}(r) .
$$

In equation (8) we have defined the unit matrix as $\hat{E}$ and used the function

$$
\hat{f}(r)=\hat{F}(\vec{r}) \exp (-\hat{\gamma} z)
$$

and the exponent with matrix in the argument is taken in sense of Taylor expansion:

$$
\exp (\hat{\gamma} z)=\sum_{n=0}^{\infty} \frac{\hat{\gamma}^{n} z^{n}}{n !}
$$

We can also use another realization of matrix exponent. Namely, it is sometimes convenient to consider it as follows:

$$
\exp (\gamma z)=\sum_{k=1}^{N} \hat{\beta}_{k} \exp \left(\eta_{k} z\right)
$$

where $\eta_{k}$ are eigenvalues of matrix $\gamma$, and symmetrical matrices $\beta_{k}$ are formed by eigenvectors of matrix $\gamma$. 


\section{Finding pair correlation functions}

To calculate the matrix $\hat{g}(r)$ from equation (8) we separately consider the cases of plane-parallel layer and infinite cylinder. In particular, we take the plane-parallel layer to be of thickness $2 L$ and the cylinder of radius $R$. Correlation functions should be zero on the boundary. So, we are looking for correlation functions in the form

$$
\begin{aligned}
& \hat{g}(\rho, z)=\sum_{m=0}^{\infty} \hat{g}_{m}(\rho) \cos \left(\frac{\pi(2 m+1) z}{2 L}\right), \\
& \hat{f}(\rho, z)=\sum_{m=0}^{\infty} \hat{f}_{m}(\rho) \cos \left(\frac{\pi(2 m+1) z}{2 L}\right)
\end{aligned}
$$

for the plane-parallel layer and

$$
\begin{aligned}
& \hat{g}(\rho, z)=\sum_{m=1}^{\infty} \hat{g}_{m}(z) J_{0}\left(\frac{\mu_{m} \rho}{R}\right), \\
& \hat{f}(\rho, z)=\sum_{m=1}^{\infty} \hat{f}_{m}(z) J_{0}\left(\frac{\mu_{m} \rho}{R}\right)
\end{aligned}
$$

for the infinite cylinder. Here we define: $J_{0}(u)$ is Bessel function and $\mu_{m}$ are zeros of Bessel function, i.e. solutions of equation $J_{0}\left(\mu_{m}\right)=0$.

For initial approximation we take a direct correlation function proportional to Dirac deltafunction, i.e. $\hat{f}(r)=\hat{C}_{(0)} \delta(\vec{r})$ and thus for harmonics $\hat{g}_{m}$ we can get the following:

$$
\triangle_{\rho} \hat{g}_{m}(\rho)-\left(\hat{\Lambda}+\frac{(2 m+1)^{2} \pi^{2}}{4 L^{2}} \hat{E}\right) \hat{g}_{m}(\rho)=-\hat{C}_{(2)}^{-1} \hat{C}_{(0)} \delta(\rho)
$$

for the plane-parallel layer and

$$
\triangle_{z} \hat{g}_{m}(z)-\left(\hat{\Lambda}+\frac{\mu_{m}^{2}}{R^{2}} \hat{E}\right) \hat{g}_{m}(z)=-\hat{C}_{(2)}^{-1} \hat{C}_{(0)} \delta(z)
$$

for the infinite cylinder. Matrix $\hat{\Lambda}$ is as follows

$$
\hat{\Lambda}=\hat{C}_{(2)}^{-1}\left(\hat{E}-\hat{C}_{(0)}+\frac{1}{4} \hat{C}_{(2)}^{-1} \hat{C}_{(1)}^{2}\right)
$$

To find harmonics $\hat{g}_{m}$ we make a Fourier transformation and thus get for the case of the system with geometry of plane-parallel layer and infinite cylinder, respectively:

$$
\begin{aligned}
& \hat{g}_{(m)}(q)=\frac{1}{L}\left[\hat{E}\left(q^{2}+\frac{(2 m+1)^{2} \pi^{2}}{4 h^{2}}\right)+\hat{\Lambda}\right]^{-1} \hat{C}_{(2)}^{-1} \hat{C}_{(0)}, \\
& \hat{g}_{(m)}(k)=\frac{1}{\pi R^{2} J_{1}^{2}\left(\mu_{m}\right)}\left[\hat{E}\left(k^{2}+\frac{\mu_{m}^{2}}{R^{2}}\right)+\hat{\Lambda}\right]^{-1} \hat{C}_{(2)}^{-1} \hat{C}_{(0)} .
\end{aligned}
$$

This enables us to present $\hat{g}_{m}$ in a more convenient way. Namely, for the plane-parallel layer we can rewrite

$$
\hat{g}_{(m)}(q)=\sum_{i=1}^{N} \frac{\hat{\lambda}_{i}}{q^{2}+q_{i}^{2}+(2 m+1)^{2} \pi^{2} / 4 L^{2}} \cdot \frac{\hat{C}_{(2)}^{-1} \hat{C}_{(0)}}{L}
$$

and

$$
\hat{g}_{(m)}(k)=\sum_{i=1}^{N} \frac{\hat{\lambda}_{i}}{k^{2}+k_{i}^{2}+\mu_{m}^{2} / R^{2}} \cdot \frac{\hat{C}_{(2)}^{-1} \hat{C}_{(0)}}{\pi R^{2} J_{1}^{2}\left(\mu_{m}\right)}
$$


can be rewritten for infinite cylinder $\left(J_{1}(u)\right.$ is the Bessel function). The above $q_{i}^{2}$ and $k_{i}^{2}(i=$ $1,2, \ldots, N)$ are eigenvalues of matrix $\hat{\Lambda}$ for the case of plane-parallel layer and cylinder, respectively. Matrices of spectral expansion can be presented as follows. Namely, we should take

$$
\hat{\lambda}_{i}=\prod_{\substack{s=1, s \neq i}}^{N} \frac{\hat{E} q_{s}^{2}-\hat{\Lambda}}{q_{s}^{2}-q_{i}^{2}}
$$

for plane-parallel layer and

$$
\hat{\lambda}_{i}=\prod_{\substack{s=1, s \neq i}}^{N} \frac{\hat{E} k_{s}^{2}-\hat{\Lambda}}{k_{s}^{2}-k_{i}^{2}}
$$

for cylinder. After inverse Fourier transformation one can get expressions for matrices of pair correlation functions. For a system with geometry of plane-parallel layer

$$
\hat{g}_{(m)}(\rho)=\sum_{i=1}^{N} \hat{\lambda}_{i} K_{0}\left(\sqrt{q_{i}^{2}+(2 m+1)^{2} \pi^{2} / 4 L^{2}} \rho\right) \cdot \frac{\hat{C}_{(2)}^{-1} \hat{C}_{(0)}}{2 \pi L},
$$

where $K_{0}(u)$ is Macdonald function. If the system has geometry of infinite cylinder, the pair correlation functions can be presented as follows:

$$
\hat{g}_{(m)}(z)=\sum_{i=1}^{N} \hat{\lambda}_{i} \frac{\exp \left(-\sqrt{k_{i}^{2}+\mu_{m}^{2} / R^{2}}|z|\right)}{\sqrt{k_{i}^{2}+\mu_{m}^{2}}} \cdot \frac{\hat{C}_{(2)}^{-1} \hat{C}_{(0)}}{2 \pi R^{2} J_{1}^{2}\left(\mu_{m}\right)} .
$$

Knowing these expressions we can easily calculate the pair correlation functions of density fluctuations.

\section{Critical parameters}

It is known [9] that the multicomponent system of $N$ components can be characterized by $N+1$ independent thermodynamic variables. The state of the system can be treated as the point in $N+1$-dimentional space. First-order phase transition hypersurface in this space is of dimension $N$ and critical hypersurface is of dimension $N-1$. The nature of critical phenomena in the system depends on the way we approach this critical hypersurface in the space of thermodynamic variables. This question is out of the bounds of this paper. Here we consider some general features of the critical state that do not depend on the particular kind of criticality.

As we can expect from studying the simple and binary finite-size systems, the space limitation causes changes in critical parameters of a multicomponent system. Indeed, critical behavior of the system is determined by parameters

$$
\kappa_{i, m}^{2}=q_{i}^{2}+\Delta_{m}^{2}
$$

where

$$
\Delta_{m}^{2}=\frac{(2 m+1)^{2} \pi^{2}}{4 L^{2}}
$$

for plane-parallel layer and

$$
\Delta_{m}^{2}=\frac{\mu_{m}^{2}}{R^{2}}
$$

for cylinder. If we compare the eigenvalue approaching zero for the finite-size system with a corresponding eigenvalue for infinite system, we can see that they differ by the value of $\Delta_{m}^{2}$. Obviously, to estimate the shift of critical parameters, we should take the minimal value from the set of different $\Delta_{m}^{2}$ i.e. take $m=0$ for plane-parallel layer and $m=1$ for cylinder. So we define

$$
\Delta_{*}^{2}=\frac{\pi^{2}}{4 L^{2}}
$$


for plane-parallel layer and

$$
\Delta_{*}^{2}=\frac{\mu_{1}^{2}}{R^{2}}
$$

for cylinder.

In the theory of critical phenomena $[1,2,17-20]$ it is generally accepted that at the close vicinity of the critical state, thermodynamic relationships are of a scaling form. According to this we assume that

$$
\kappa^{2}=r_{0}^{-2}\left(\frac{X-X_{\mathrm{C}}}{X_{\mathrm{C}}}\right)^{2 \nu}
$$

where $\kappa^{2}$ means leading to zero eigenvalue, $\nu$ is critical index (its value depends on the way we fix thermodynamic parameters [9]), $X$ is thermodynamic parameter and $X_{\mathrm{C}}$ is its critical value (for infinite system), $r_{0}$ is amplitude of correlation length. Then, we can estimate the shift of critical value $X_{\mathrm{C}}$ due to space limitation. Namely, we can get for critical value $X_{\mathrm{C}}^{f s}$ of the finite-size system

$$
X_{\mathrm{C}}^{f s}=\frac{X_{c}}{1+\left(\Delta_{*} r_{0}\right)^{1 / \nu}} \sim h^{-1 / \nu},
$$

where $h$ is linear size of the system. This result is in good agreement with the available theoretical and experimental data $[1,2,9,10]$.

\section{Conclusions}

We have found the expressions for pair correlation functions of density fluctuations for finitesize multicomponent liquid (with geometry of plane-parallel layer and infinite cylinder) with the external field that is applied to the system. Any of these correlation functions can be presented as a linear combination of a fixed number of basic functions. This concept can be very fruitful if used in handling the experimental data.

The presence of external field results in exponential multipliers in the expressions for pair correlation functions. This leads to an increase or decay of fluctuations along the field or in the opposite direction, respectively. Nevertheless, it is possible to show that even under these conditions, the pair correlation functions still remain short-ranged far from critical region.

We have also shown that in the case of finite-size multicomponent system, the space limitation causes a shift of critical parameters. The dependence of shifts on linear size of the system is of a scaling-law form. This result is in close agreement with the prediction of isomorphism hypothesis $[1,8]$ and at least does not contradict the available data on critical behavior of binary mixtures (both spatially infinite and finite-size). Nevertheless, one should remember that this conclusion is made based on some special assumptions about the dependence of eigenvalues on thermodynamic parameters of the system. All this imposes some restrictions on our results. On the other hand, our results in limiting cases, i.e. for simple and binary systems (spatially infinite and finite-size), give a well-known formulae that was previously received for these systems in numerous works (see for example $[1,6,7,21])$ and we can conclude that they are absolutely realistic.

\section{References}

1. Anisimov M. Critical Phenomena in Liquids and Liquid Crystals. Moskow, Nauka, 1987 (in Russian).

2. Patashinskii A.Z., Pokrovskii V.L. The Fluctuation Theory of Phase Transitions. Oxford, Pergamon Press, 1979.

3. Yukhnovskii I.R. Phase Transitions of the Second Order. The Collective Variables Method. Kiev, Nauk. Dumka, 1985 (in Russian).

4. Privman V. (ed.) Finite Size Scaling and Numerical Simulation of Statistical Systems. Singapore, World Scientific, 1990.

5. Fisher M.E., Critical Phenomena. Proc. 51st "Enrico Fermi" Summer School, Varenna, Italy, ed. M.S. Green. Academic Press, NY, 1971.

6. Chalyi A.V., Chalyi K.A., Chernenko L.M., Vasil'ev A.N., Cond. Mat. Phys., 2000, 3, 335. 
7. Chalyi A.V., Chernenko L.M. Phase Transitions In Finite-Size Systems and Synaptic Transmission. In: Dynamical Phenomena at Interfaces, Surfaces and Membranes. New York, 1993, p. 457-464.

8. Fisher M.E., Phys. Rev., 1968, 176, 257.

9. Griffiths R.B., Wheeler J.C., Phys. Rev. A, 1970, 2, 1047.

10. Bellocq A.M., Gazeau D., J. Phys. Chem., 1990, 94, 8933.

11. Gauter K., Peters C.J., Fluid Phase Equilibria, 1998, 150-151, 501.

12. Gauter K., Heidemann R.A., Peters C.J., Fluid Phase Equilibria, 1999, 158-160, 133.

13. Kolar P., Kajima K., Fluid Phase Equilibria, 1996, 118, 175.

14. Vasiliev A., Theoretical and Mathematical Physics (transl. from rus.), 2003, 135, 714.

15. Stenley H. Introduction To Phase Transitions And Critical Phenomena. Clarendon Press, Oxford, 1971.

16. Zubarev D.N. Nonequilibrium Statical Thermodynamics. Consultant Bureau. New-York, 1974.

17. Cardy J.L. (ed.) Finite-Size Scaling. North Holland, Amsterdam, 1988.

18. Wilding N.B., J. Phys.: Condens. Matter, 1997, 9, 585.

19. Binder K., Ann. Rev. Phys. Chem., 1992, 43, 33.

20. Fisher M.E., Rev. Mod. Phys., 1998, 70, 653.

21. Chalyi A., J. Mol. Liquids, 1993, 58, 179.

\title{
Критичні параметри та парні кореляції в просторово обмежених багатокомпонентних рідинах
}

\author{
О.М. Васильєв ${ }^{1}$, О.В. Чалий ${ }^{1,2}$ \\ 1 Київський університет ім. Т.Шевченка, \\ фізичний факультет, кафедра теоретичної фізики, \\ просп. Глушкова 2, корпус 1, Київ, 03680, Україна \\ 2 Кафедра Фізики національного \\ медичного університету \\ ім. акад. О.О. Богомольця, \\ бульв., Шевченка 13, Київ 01601, Україна \\ Отримано 3 серпня 2005 р., в остаточному вигляді 20 лютого 2006 р. \\ Досліджується багатокомпонентна просторово обмежена рідка система з геометрією плоского па- \\ ралельного прошарку та циліндру. Системи є анізотропними внаслідок дії зовнішнього слабкого \\ поля. Для таких систем знаходяться парні кореляційні функції та аналізується вплив просторового \\ обмеження та зовнішнього поля на кореляційну поведінку багатокомпонентної рідини. Результати \\ порівнюються з тими, що наявні для простих та бінарних просторово обмежених систем.
}

Ключові слова: кореляційна функція, критичний стан, багатокомпонентна рідина

PACS: $05.70 . F h, 05.70 . J k, 64.60 . F r, 68.35 . R h$. 